\title{
Nota sobre el crecimiento de Crocodylus acutus en cautiverio.
}

Fabio G. Cupul-Magaña, Helios Hernández-Hurtado.

Departamento de Ciencias, Centro Universitario de la Costa, Universidad de Guadalajara. Puerto Vallarta, Jalisco, México.

El crecimiento puede ser definido como una modificación irreversible, asociada con la actividad fisiológica del protoplasma y, comúnmente, acompañada por un incremento irreversible en el volumen o masa celular. Éste es un atributo esencial del material vivo, mismo que puede ser afectado directamente por los nutrimentos que son asimilados y por las condiciones del hábitat donde se desarrolla el ser vivo (1).

Al respecto de la actividad fisiológica; el sistema digestivo de los cocodrilos es admirable por varias razones. Primero, el estómago es el de mayor acidez dentro del grupo de los vertebrados, lo que le permite digerir casi la totalidad de lo que consume. Segundo, cerca del $60 \%$ de la energía contenida en la comida que ingiere es almacenada en forma de grasa, en la cola, en órganos mesentéricos del abdomen, a lo largo del dorso y casi en cualquier parte del cuerpo. Incluso, una fracción de la energía contenida en las proteínas puede ser convertida a grasas (2).

Lo anterior, les permite a estos reptiles sobrevivir largos períodos de tiempo sin alimento. Por ejemplo, una cría de cocodrilo puede vivir alrededor de cuatro meses sin probar alimento, ya que hace uso de la grasa contenida en el saco de yema remanente que se localiza bajo el abdomen. Por su parte, a un gran cocodrilo, de aproximadamente media tonelada de peso, le basta con alimentarse una vez cada dos años para resolver sus problemas de sobrevivencia (2).

Claro está, que el eficiente metabolismo de los cocodrilos tiene su costo. Uno de los más relevantes es el relacionado con la tasa de crecimiento; ya que cuando el cocodrilo recibe una ración constante de alimento (generalmente cuando es criado en cautiverio) puede crecer alrededor de medio metro en un año, mientras que en el medio salvaje su tasa de crecimiento es mucho menor. Por su parte, como las crías almacenan energía en forma

Solicitud de sobretiros: M. en C. Fabio G. Cupul-Magaña. Depto. de Ciencias, Centro Universitario de la Costa, Universidad de Guadalajara, Av. Universidad de Guadalajara No. 203, Delegación Ixtapa, C.P. 48280, Puerto Vallarta, Jalisco, México. $\quad$ Email: fcupul@pv.udg.mx 


\section{FG Cupul-Magaña, H Hernández-Hurtado.}

de grasa, si pasan por un período largo de inanición, parte de sus recursos energéticos son directamente destinados a incrementar la talla y la masa muscular (2).

Con relación al crecimiento del cocodrilo de río (Crocodylus acutus), es pobre la información generada hasta el momento. Al respecto, se tiene el trabajo en cautividad realizado en Chipas; en el cual, ejemplares de $27.0 \mathrm{~cm}$ de longitud, lograron crecer hasta $4.0 \mathrm{~cm}$ por mes (3). En Florida, Estados Unidos, ejemplares silvestres de cocodrilo de río crecieron un máximo de $6.42 \mathrm{~cm}$ por mes, durante los primeros 80 días de vida; asimismo, lograron crecer hasta $3.36 \mathrm{~cm}$ por mes, en los primeros 17 meses de vida (4). En Cuba, se ha probado satisfactoriamente una dieta a base de productos marinos y cárnicos, enriquecida con un suplemento que incluye hemolizado como antianémico, Tetramisol como antiparasitante, Cloranfenicol como antibiótico, Dextrosa al 5\% y azúcar cruda como energético y polivitaminas; con esta dieta, han logrado que los ejemplares alcancen tallas promedio de $70.9 \mathrm{~cm}$ en su primer año de vida (5). Para el grupo de los cocodrilos, se ha establecido un crecimiento promedio mensual de $4.6 \mathrm{~cm}$ en los dos primeros años de vida (6).

En el cuadro 1, los autores presentan los resultados de crecimiento observado, entre 1995 y 1997 , en 4 ejemplares adultos y 13 crías de cocodrilo de río, cautivas en las instalaciones del Rancho Ecológico El Quelele, Nayarit; distante 15 $\mathrm{km}$ al norte de la ciudad de Puerto Vallarta, Jalisco. Los organismos adultos y crías se confinaron a dos acuaterrarios con superficies de 1,604 $\mathrm{m}^{2}$ y $21 \mathrm{~m}^{2}$,

Cuadro 1

Datos de crecimiento mensual (en cm) de longitud total de cocodrilo de río, entre 1995 y 1997, en el Rancho Ecológico El Quelele, Nayarit. Nota: los cuadros con guión representan información no registrada.

\begin{tabular}{|c|c|c|c|c|c|c|c|c|c|c|c|}
\hline $\begin{array}{c}\text { No. } \\
\text { ejemplar }\end{array}$ & $\begin{array}{c}\text { Mar } \\
96\end{array}$ & Abr & May & Jun & Jul & Ago & Sep & Oct & Nov & Dic & $\begin{array}{c}\text { Ene } \\
97\end{array}$ \\
\hline 1 & 43.7 & 47.0 & 52.0 & 55.9 & 60.1 & 65.0 & 72.6 & - & - & - & 78.4 \\
\hline 2 & 48.9 & 53.1 & 56.4 & 58.3 & 58.9 & - & - & - & - & - & - \\
\hline 3 & 42.8 & 47.5 & 51.5 & 55.4 & 58.9 & 62.5 & 69.5 & - & - & 74.2 & 74.2 \\
\hline 4 & 44.5 & 44.7 & 48.8 & 53.0 & 57.2 & 62.0 & 68.0 & - & - & 72.0 & 72.8 \\
\hline 5 & 44.8 & - & - & 52.8 & 56.8 & 62.0 & 68.5 & - & - & 70.9 & 71.8 \\
\hline 6 & 43.2 & 47.0 & 51.8 & 56.4 & 60.0 & 65.0 & 70.8 & - & - & 76.7 & 77.0 \\
\hline 7-macho & 198.0 & - & - & - & - & - & 212.0 & - & - & - & - \\
\hline 8 & - & - & - & 77.3 & 77.3 & 77.5 & 79.9 & - & - & 86.8 & 88.2 \\
\hline 9 & 45.3 & 47.6 & 52.0 & 57.1 & 63.8 & 68.8 & 72.1 & - & - & 78.1 & 78.8 \\
\hline 10 & - & - & - & - & - & 85.0 & 87.3 & - & - & 90.9 & 92.2 \\
\hline 35-macho & 126.0 & - & - & - & - & - & - & - & - & - & - \\
\hline \multirow[t]{2}{*}{ s/n-macho } & - & - & - & - & - & - & 194.0 & - & - & - & 194.0 \\
\hline & $\begin{array}{l}\text { Jul } \\
95\end{array}$ & Ago & Sep & Oct & Nov & Dic & $\begin{array}{l}\text { Ene } \\
96\end{array}$ & Feb & Mar & Abr & May \\
\hline 402 & - & - & - & - & 85.0 & 85.0 & - & - & 86.0 & 86.3 & - \\
\hline A1 & 29.5 & 30.6 & 32.1 & 34.6 & 36.3 & 36.3 & - & - & - & - & - \\
\hline B2 & 29.0 & 29.4 & 30.4 & 30.8 & 30.9 & - & - & - & - & - & - \\
\hline $\mathrm{C} 3$ & - & - & - & - & 86.0 & 86.1 & - & - & - & - & - \\
\hline D4-macho & - & - & - & - & - & 187.0 & - & - & - & - & - \\
\hline
\end{tabular}

\section{Revista Biomédica}


Crecimiento de Crocodylus acutus en cautiverio.

respectivamente.

De manera general, la alimentación de los adultos fue a base de trozos grandes de carne fresca de res, pescado y aves de corral. A las crías se les alimentó principalmente con pequeños trozos de pescado fresco; aunque, en ocasiones, se les proveyó de camarones de río pequeños; además, frecuentemente se encendía por la noche, dentro del acuaterrario, un foco de 60 watts, el cual atraía insectos para que fueran atrapados y consumidos por las crías.

El suministro de alimento se realizaba dos veces por semana y la ración de comida se estableció de acuerdo con el peso corporal de los ejemplares; siendo del 20 al $26 \%$ del peso corporal en las crías, del 12 al $15 \%$ en juveniles y del $11 \%$ en adultos (6).

Los resultados sobresalientes de este trabajo, mostraron que el índice promedio mensual de crecimiento en las crías (para esta operación, sólo se tomaron los datos de los ejemplares $1,3,4,5$, 6 y 9) fue de $3.14 \mathrm{~cm}$; lo que representó un incremento promedio mensual del $7.12 \%$ en relación a la talla inicial de los ejemplares. Con respecto al ejemplar adulto marcado con el número 7, que inicialmente midió $1.94 \mathrm{~m}$, transcurridos seis meses, tuvo un aumento de $2.33 \mathrm{~cm}$ por mes; es decir, sólo aumentó el $1.17 \%$ mensual de su longitud total inicial. Esta información coincide con los resultados obtenidos en otros trabajos (6); además, dio pie para establecer que la superficie de los acuaterrarios, la densidad de individuos por área y la temperatura, se encuentran relacionados con el crecimiento ${ }^{7}$.

Palabras clave: Crocodylus acutus, crecimiento, cautiverio, dieta.

\section{REFERENCIAS.}

1.- Scott TA. Concise Encyclopedia: Biology. Berlin: Walter de Gruyter; 1995. p. 1287.

2.- Garnett S. Efficient metabolism. En: Ross CA, Garnett S, editores. Crocodiles and alligators. New York: Facts on
File; 1989. p. 84.

3.- Álvarez del Toro M. Los Crocodylia de México. México: IMERNAR; 1974. p. 70.

4.- Throbjarnarson JB. Ecology of the american crocodile, Crocodylus acutus. En: Crocodiles their ecology, management and conservation. IUCN Publication New Series. p. 228-259.

5.- Benavides-Calvo RR, Fonseca-Sanz RN. Enriquecimiento dietético y su efecto sobre el crecimiento del Crocodylus acutus en el zoocriadero de Manzanillo. Cuba: Empresa Nacional para la Conservación de la Flora y la Fauna; 1999. p. 11.

6.- Bolton M. La explotación del cocodrilo en cautividad. Italia: Guía FAO Conservación 22; 1994. p. 156.

7.- Hernández-Hurtado H. Recomendaciones para el desarrollo de un centro de acopio de cocodrilos en el Rancho Ecológico El Quelele, Municipio de Bahía de Banderas, Nayarit [Tesis licenciatura]. Guadalajara (Jalisco, México): Universidad de Guadalajara; 1997. 